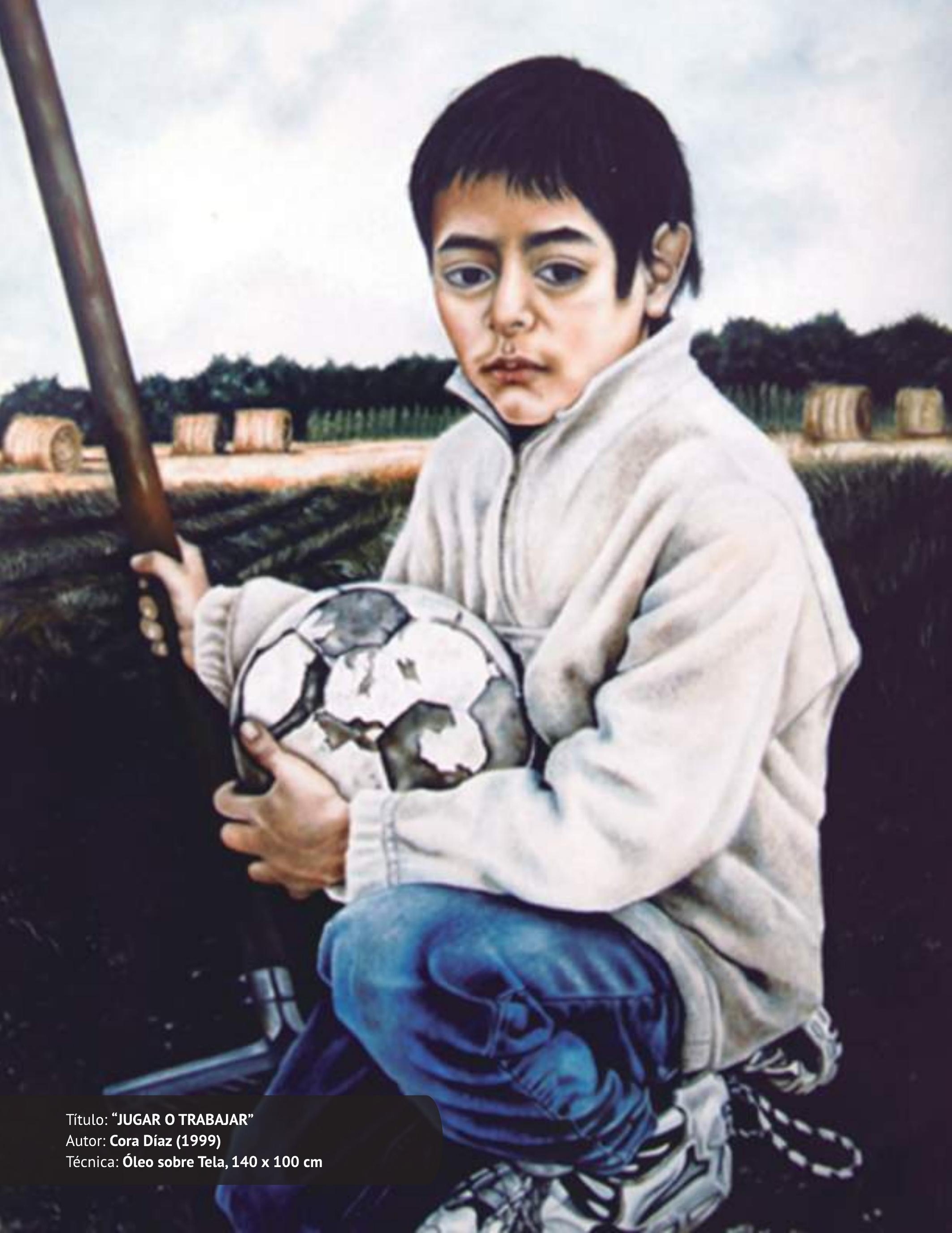




\section{ESTUDIO DE RESPONSABILIDAD SOCIAL, MONITOREO DE HOMICIDIOS EN MÉXICO}

SOCIAL RESPONSIBILITY STUDY, HOMICIDE MONITORING IN MEXICO

\section{PAULA PATRICIA BORREGO GARZA}

\section{RESUMEN}

Este trabajo presenta al Monitoreo Nacional de Homicidios como una manera de medir el pulso a la inseguridad en México a través del control estadístico de procesos. Este monitoreo se alimenta con los datos publicados diariamente en el sitio oficial del Gobierno Federal. Nuestra intención es brindarle al público en general las herramientas para que, sin ser expertos en el tema de homicidios, puedan consumir, contextualizar y juzgar la información. Se encuentra estacionalidad en el fenómeno de los homicidios, que registran mayores números los fines de semana. Se logra dar lectura a los datos dividiendo el monitoreo en periodos cortos de tiempo, sin embargo, se observa dinamicidad en los datos, por lo que esta investigación sigue en curso. Se propone ser consumidores directos de la información, apoyándonos en los monitoreos para poner los datos en el contexto de su historia reciente, en contraste de informarnos de noticias con juicios subjetivos. Con base a los datos, podremos evaluar las acciones de las autoridades.

PALABRAS CLAVE: HOMICIDIOS, SEGURIDAD, ESTADÍSTICA, MONITOREO, CONTROL, GOBIERNO.

\section{ABSTRACT}

In this paper we introduce the National Homicide Monitoring as a way to gauge the safety issues in Mexico, through statistical process control. This Monitoring is fed with the data published by the Federal Government in their official website. Our intention is to provide to the general public the tools so, without being experts in the homicide topic, we can consume, put in context and judge the information. We found seasonality in the homicide phenomena, the records show higher data on the weekends. We were able to read the monitoring, by taking little segments in time, nevertheless, due to the dynamicity of the data, this research is still in course. It is proposed to consume the raw data, with the help of the monitoring, to give context to the numbers by putting them side to side with its recent history instead of informing ourselves through judgmentally subjective news. With this, we can evaluate the actions of the authorities based on such data.

KEYWORDS: HOMICIDES, SAFETY, STATISTICS, MONITORING, CONTROL, GOVERNMENT. 
C omo estudiante y egresada de la Universidad Autónoma de Nuevo León siempre encontré fascinante nuestro lema: Alere Flammam Veritatis o Alentando la Flama de la Verdad. Hoy toca llevarlo a la práctica. En tiempos de noticias falsas, hechos alternativos, videos virales, audios por mensajería instantánea, entre otros, se hace obligatorio siempre verificar las fuentes de las cuales nos informamos.

En el tema de la inseguridad, nos enteramos de incidentes que nos dejan desconcertados y desmoralizados, en pocas palabras. Vivimos y padecemos la realidad de nuestro entorno. Pero, ¿Qué pasa en el contexto nacional? ¿Cómo sabemos si hay avances o retrocesos en este renglón que tanto ha flagelado a nuestro país?

Empezó el sexenio del presidente López Obrador con cuestionamientos en esta materia. Decidió, por primera vez en la historia, hacer públicos los datos de homicidios dolosos en el país en el sitio oficial del Gobierno Federal (Gobierno de México, 2019).

Nos dimos a la tarea de analizar esos datos desde que se empezaron a publicar. Nuestra intención es brindarle al público en general las herramientas para que, sin ser expertos en el tema de homicidios, podamos consumir, contextualizar y juzgar la información.

A continuación, presento el Monitoreo Nacional de Homicidios como una manera de medir el pulso a la inseguridad en México, a través de herramientas estadísticas efectivas y a la vez sencillas para visualizar los datos y entenderlos desde esta pers-

El Monitoreo Nacional de Homicidios como una manera de medir el pulso a la inseguridad en México, a través de herramientas estadísticas efectivas y a la vez sencillas para visualizar los datos y entenderlos desde esta perspectiva

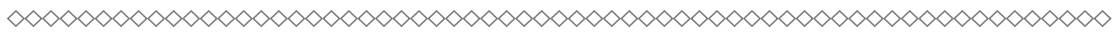

pectiva. Al terminar este artículo, podrá tener una lectura de la situación actual del país en cuestión de seguridad y podrá darle seguimiento cada semana a través de nuestras redes.

\section{METODOLOGÍA}

De la estadística se desprende el Control Estadístico de Procesos, siendo el corazón de esta rama los diagramas de control. Las aplicaciones más comunes de estas técnicas y análisis se encuentran en las líneas de producción de la industria, en donde la variabilidad se traduce como falta de calidad que se necesita controlar y minimizar.

Se entiende por control el mantener un proceso en operación estable y predecible. El propósito del control es simple:

Una vez que se ha mejorado y se han documentado los avances, se continúa midiendo la evolución del proceso rutinariamente y se ajusta si es necesario (Pande, Neuman and Cavanagh, p. 346, 2000).

Salvador Borrego, doctor egresado de la UANL y quien preside esta investigación, ha aplicado por 20 años los monitoreos estadísticos al área social con el propósito fundamental de estudiar la variación que en ésta tienen ciertos procesos.

\section{¿QUÉ ES UN MONITOREO?}

Un monitoreo va recopilando la información de un proceso en un diagrama que consta de 5 límites: La línea central que representa el promedio y los límites de advertencia y alerta que representan la variación esperada de los datos, en sus siguientes modalidades (Pande, Neuman and Cavanagh, 2002).

- Advertencia Positiva

- Advertencia Negativa

- Alerta Positiva

- Alerta Negativa

En la Gráfica 1 (pág. 57) presentamos el Monitoreo Semanal de Homicidios, para describir las herramientas antes comentadas.

\section{COLORES DEL MONITOREO}

Para visualizar mejor el monitoreo, utilizamos los siguientes colores:

- Negro: Límite Central

- Rojo: Límite de Alerta Negativa

- Naranja: Límite de Advertencia Negativa

- Verde: Límite de Advertencia Positiva

- Azul: Límite de Alerta Positiva 


\section{GRÁFICA 1. Monitoreo Semanal de Homicidios}
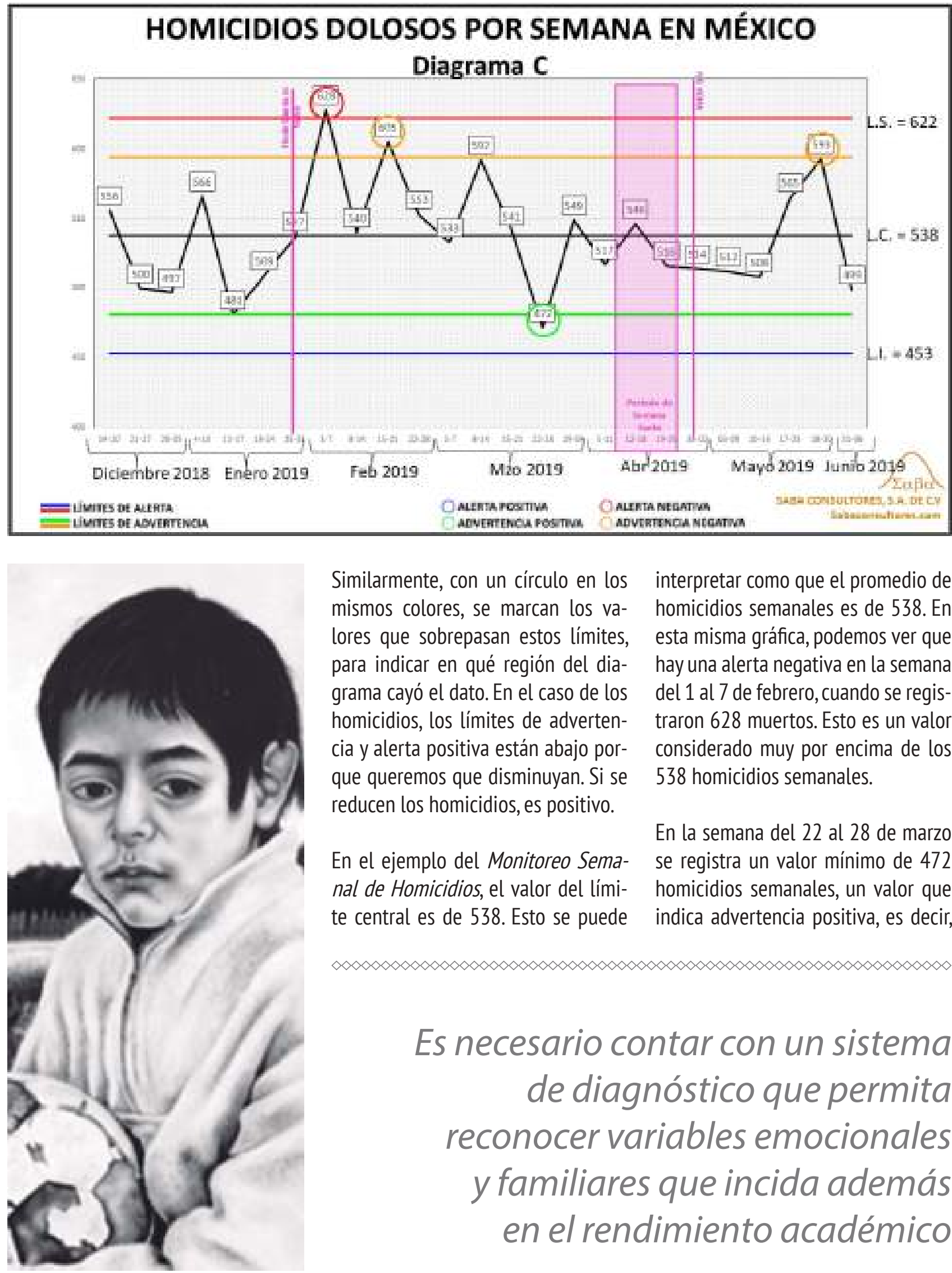

Similarmente, con un círculo en los mismos colores, se marcan los valores que sobrepasan estos límites, para indicar en qué región del diagrama cayó el dato. En el caso de los homicidios, los límites de advertencia y alerta positiva están abajo porque queremos que disminuyan. Si se reducen los homicidios, es positivo.

En el ejemplo del Monitoreo Semanal de Homicidios, el valor del límite central es de 538. Esto se puede interpretar como que el promedio de homicidios semanales es de 538 . En esta misma gráfica, podemos ver que hay una alerta negativa en la semana del 1 al 7 de febrero, cuando se registraron 628 muertos. Esto es un valor considerado muy por encima de los 538 homicidios semanales.

En la semana del 22 al 28 de marzo se registra un valor mínimo de 472 homicidios semanales, un valor que indica advertencia positiva, es decir,

\section{Es necesario contar con un sistema de diagnóstico que permita reconocer variables emocionales y familiares que incida además en el rendimiento académico}


que disminuyeron significativamente los homicidios. En color magenta se marcan eventos que pueden tener incidencia ya sea para bien o para mal en lo que a inseguridad se refiere.

\section{LECTURA DEL MONITOREO}

Si un valor se encuentra entre los límites de advertencia se considera estable, es decir, las variaciones que se registran son típicas. Walter $A$. Shewhart, el padre del control de calidad, denomina a esto como causas azarosas.

Todo proceso tiene cierta variabilidad, resultado del acumulamiento de pequeñas variaciones que provienen de una gran cantidad de fenómenos que influyen sobre el proceso constantemente (Montgomery, 2009). La variación que rompe con la estabilidad comúnmente es generada por un fenómeno aislado pero de gran magnitud (Borrego, 2010).
El Sistema de Alertas y Advertencias nos sirve para saber cuándo un valor se está saliendo de los parámetros esperados. Es posible tener alertas 0 advertencias (puntos fuera de los límites de control) de manera aislada, éstas indicarán valores que rompieron el equilibrio y perdieron la estabilidad. Sin embargo, si éstas ocurren con cierta frecuencia, entonces son señales de que la realidad del proceso está cambiando.

\section{SERIES Y TENDENCIAS}

Si 8 valores consecutivos se encuentran por encima del límite central (negro) se indica una serie por encima del promedio y se recalculan los 5 límites para ajustarnos a la nueva realidad. Si se registran 8 valores consecutivos por debajo del límite central, le llamamos serie por debajo del promedio y también recalculamos los límites del diagrama. Al registrarse 8 valores consecutivos en incremento de manera consistente, tenemos lo que se conoce como una tendencia al alza. Si los valores van decreciendo, entonces será una tendencia a la baja.

Todo lo anterior es una descripción somera del funcionamiento y lectura de los monitoreos. Detrás de estos, hay todo un potentado de la ciencia estadística. Para realizar una futura discusión:

- Los homicidios de un día a otro se asumen independientes.

- El diagrama C, creado por Walter A. Shewhart, es utilizado para el número de eventos no deseados, ocurridos en una misma unidad de tiempo.

- El fenómeno, por sus características, se representa con una distribución de Poission.

- Los límites de advertencia están calculados a 2 sigmas.

- $\quad$ Los límites de alerta a 3 sigmas.

\section{GRÁFICA 2. Monitoreo Nacional de Homicidios}

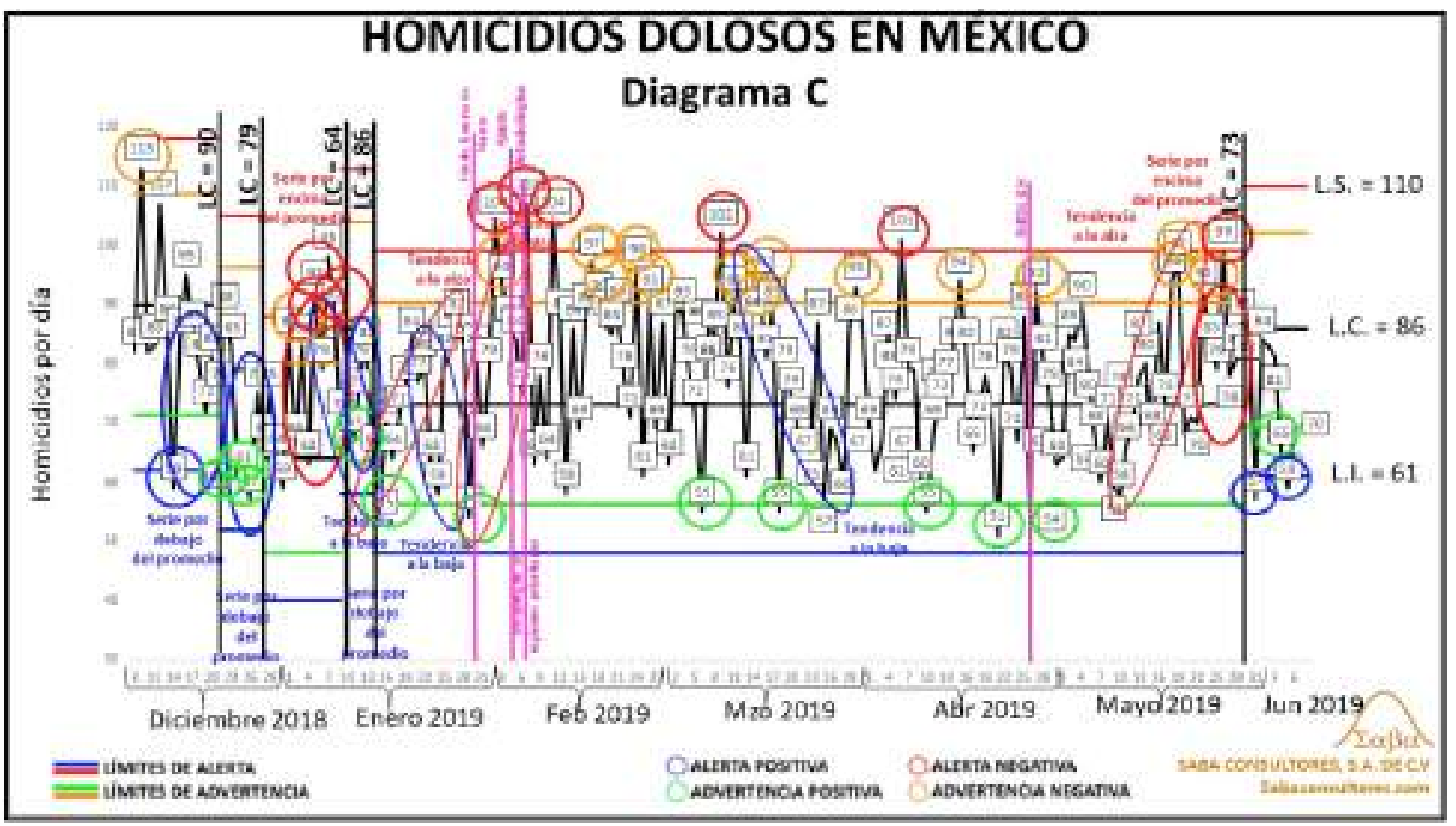




\section{GRÁFICA 3. Seguimiento Cotidiano del Monitoreo}

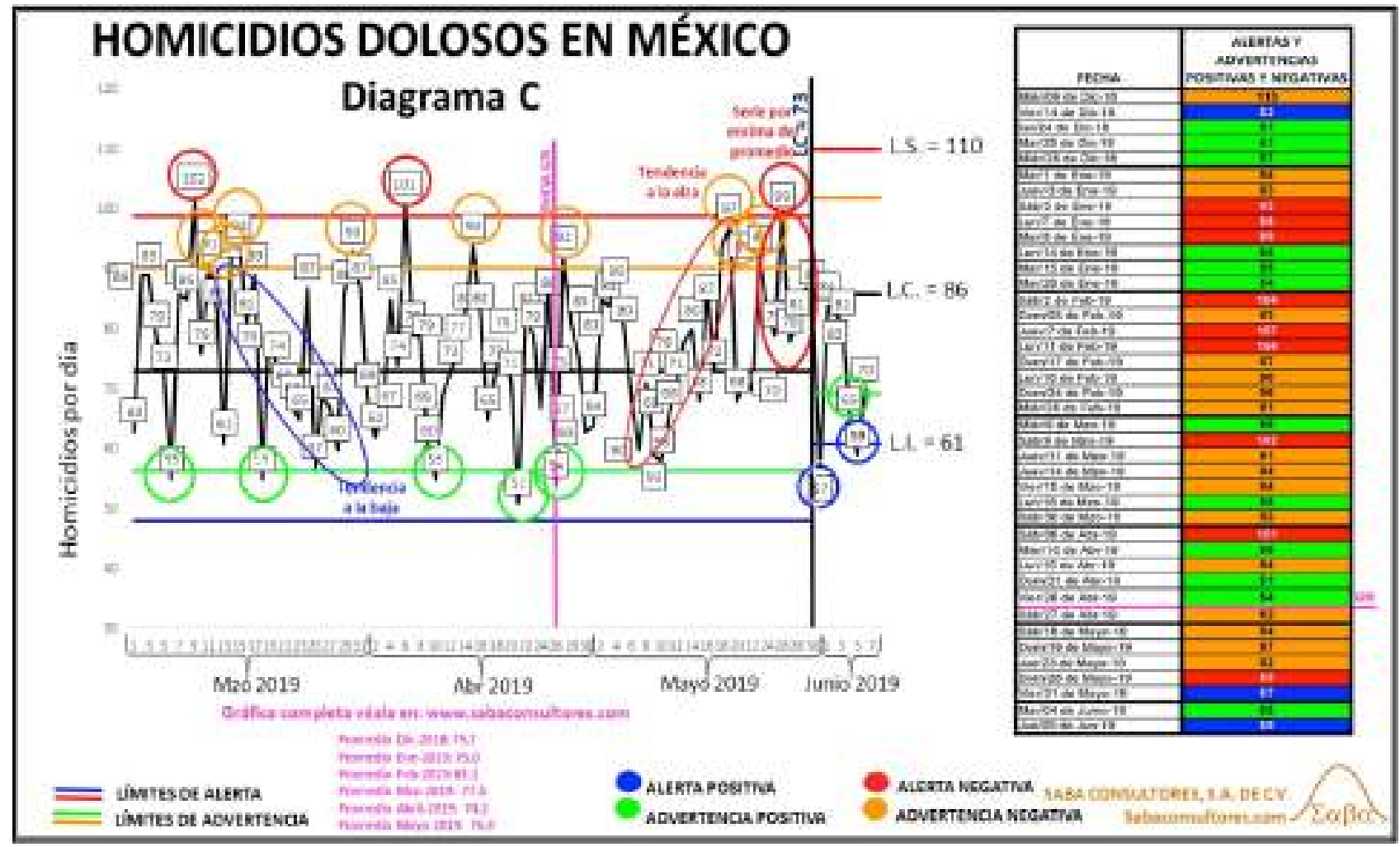

\section{RESULTADOS}

Ahora que podemos leer los monitoreos, les presentamos el Monitoreo Nacional de Homicidios generado diariamente. La Gráfica 2 (pág. 58) contiene mucha información y puede ser abrumadora, pero empecemos desmenuzándola para comprender la situación de los homicidios diarios.

\section{LÍMITES CENTRALES}

Como se mencionó en la sección anterior, cuando hay 8 puntos por encima o por debajo del límite central se recalculan los límites. Cuando esto sucede, se marca una línea vertical negra y se anota el anterior límite central como referencia. Al inicio de este monitoreo, el límite central era de 90 homicidios por día. Después cambió a 79, 64, 86 y se mantuvo en 73 por más de 4 meses, hasta recientemente regresar a 86 .

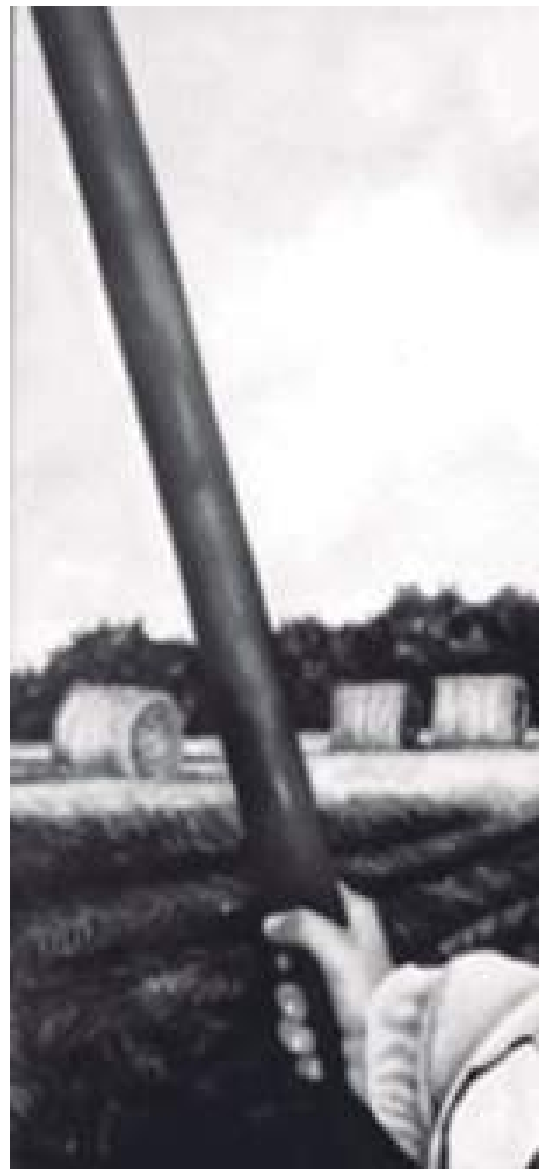

Cuando inició el monitoreo, se cambiaba el límite central cada 5 puntos por encima o por debajo del promedio. Anteriormente no se conocía el fenómeno de los homicidios, por lo que se hizo un ajuste a cada 8 registros por encima o por debajo del promedio. Esta técnica aunque rigurosa en su estadística-matemática para definir los límites de control, es flexible en cuanto a los criterios para ajustar el proceso.

\section{SEGUIMIENTO COTIDIANO DEL MONITOREO}

Acerquémonos más a las alertas y advertencias a través de la Gráfica 3. Esta es la gráfica anterior recortada a los últimos tres meses. A la derecha se muestra una tabla con el día y valor de las alertas y advertencias en ambos sentidos. Al dar lectura a esta gráfica podemos ver la historia en el 


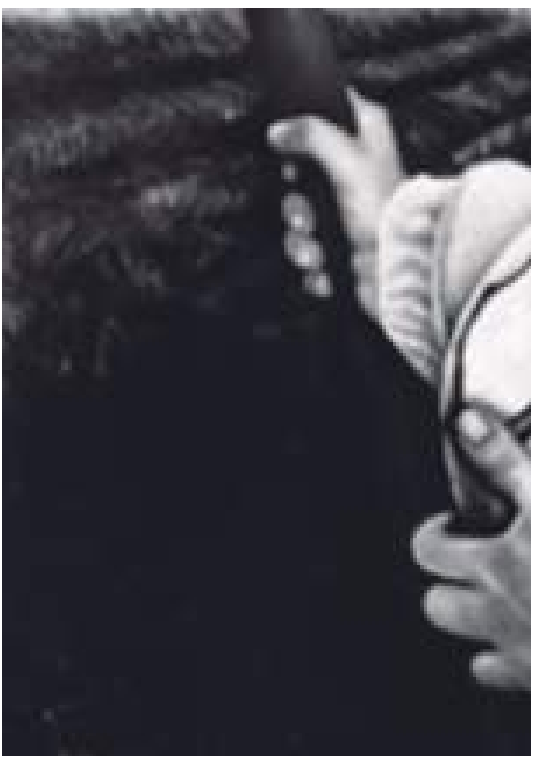

tiempo del fenómeno de los homicidios, qué valores estuvieron en la zona de estabilidad y qué valores se salieron de esta.

Podemos ver de manera nítida una tendencia a la baja que se prolonga por toda la segunda mitad de marzo y una tendencia al alza que inicia el 9 de mayo y termina el 20 de mayo. También vemos cómo se forma una serie por encima del promedio a finales del mes.

El hecho de que los valores estén entre los límites de control, ¡No significa que se están dando buenos resultados! Simplemente, significa que esta herramienta que usamos captura efectivamente la variabilidad de los datos (Pande, Neuman and Cavanagh, 2002).

También me gustaría agregar que los diagramas de control son tan buenos como la calidad de los datos. Se recomienda a las personas encargadas de recopilar datos, implementar un proceso de validación y confiabilidad de los mismos (Pande, Neuman and Cavanagh, 2002).

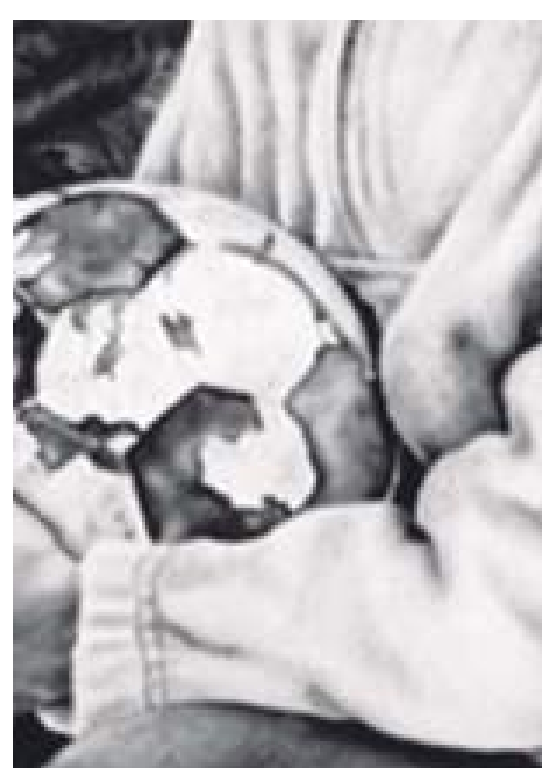

\section{CONCLUSIONES Y DISCUSIÓN}

Con base en la información anterior podemos concluir las siguientes 3 observaciones:

1. Hay un patrón de estacionalidad que se observa: los homicidios incrementan los fines de semana. La mayoría de las alertas y advertencias negativas se presentan en sábados o domingos.

2. El fenómeno de los homicidios lo podemos evaluar según la sección en el tiempo que se busque analizar. Al día de hoy da señales contradictorias pues a pesar de que recientemente se incrementaron los límites (pasando el límite central de 73

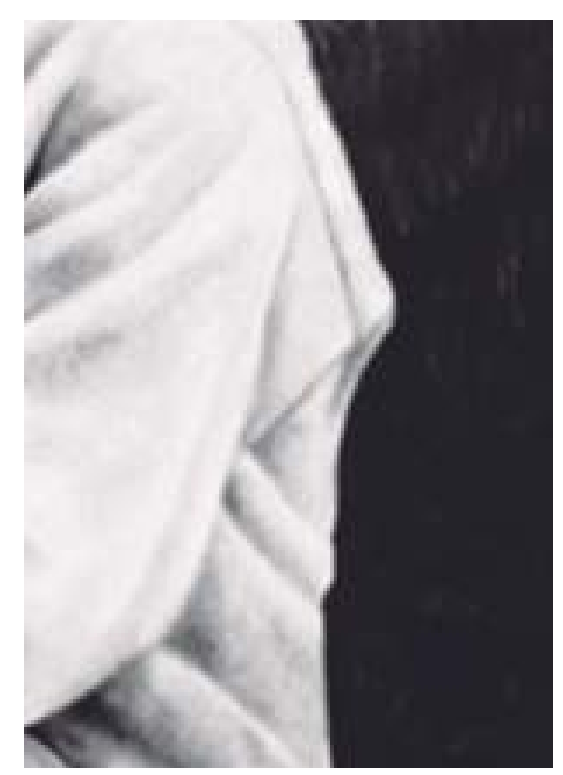

homicidios diarios en promedio a 86) tenemos signos positivos al registrarse una advertencia y una alerta positiva.

3. La segunda observación podría cambiar en cualquier momento, pues esto es un fenómeno dinámico, no estático.

Ya que mencionamos que la aplicación principal de esta herramienta es en la industria, imaginemos a las autoridades de seguridad pública como empleados de una línea de producción que estén al pendiente de estos diagramas para ver su desempeño. Los altos mandos buscarían que el proceso sea por lo menos estable $y$,

\section{Los diagramas de control son tan buenos como la calidad de los datos. Se recomienda a las personas encargadas de recopilar datos, implementar un proceso de validación y confiabilidad de los mismos}




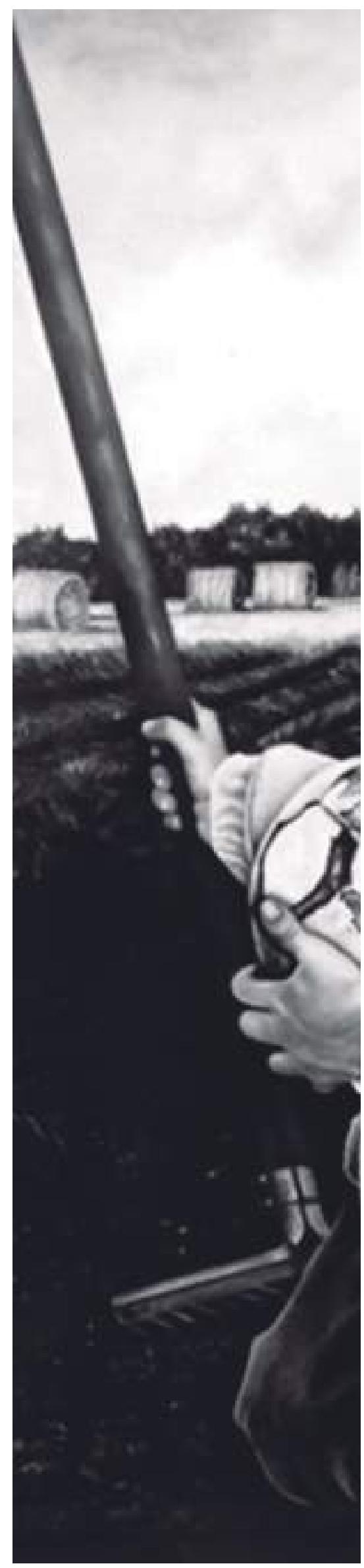

\section{En el presente sexenio, sí contamos con esta información, sin embargo no se contextualiza en un diagrama de control}

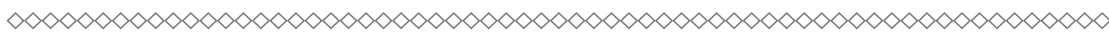

por supuesto, que disminuya el número de homicidios. Si reciben una alerta positiva, verán qué hicieron bien ese día para obtener ese resultado. Si reciben una alerta negativa, evaluarán en qué se falló y qué se puede hacer para evitar que se repita.

En el sexenio del presidente Felipe Calderón, se criticó mucho su estrategia de seguridad por parte de los medios y los ciudadanos. Pero a pesar de las alarmantes cifras de muertos, desaparecidos y encontrados en fosas, nunca se conoció la evolución del fenómeno.

En el presente sexenio, sí contamos con esta información, sin embargo no se contextualiza en un diagrama de control. Esto hace que los análisis que se desprenden de ésta sean subjetivos cuando, por ejemplo, se comparan las cifras con aquéllas de años anteriores o de inicios de sexenios anteriores.

Actualmente, no tenemos conocimiento de alguien más que utilice la información de homicidios del gobierno federal para hacer análisis serios y apartidistas. Ciudadanos, medios de comunicación, asociaciones civiles y grupos de investigadores, conviven en una atmósfera de polarización que hace difícil mantener la neutralidad, si es que se aventuran a hablar de ello.
Nosotros no estamos exentos de semejante ambiente. Pero conocemos a cabalidad la herramienta que les presentamos en esta ocasión, que tiene la virtud de ayudarnos a digerir los datos duros al contrastarlos con la historia reciente de los mismos.

Lo anterior es precisamente la aportación de esta investigación. Brindar al ciudadano, al medio de comunicación 0 asociación que lo desee, información veraz para los propósitos que lo ocupen.

En el contexto académico aprendimos a citar las fuentes, pero actualmente también se nos pide cuestionar si las fuentes son confiables. ¿Quién sería una fuente confiable para darnos información en seguridad? ¿El presidente? ¿Las autoridades? 0 por qué no, nosotros mismos consultando los datos duros.

Como ciudadanos, nos encontramos ya muy sensibilizados en el tema de seguridad. Con esta herramienta a la mano, ahora sí podremos evaluar a ciencia cierta las acciones de las autoridades. Mantengamos como parte de nuestra información los datos duros nacionales en el contexto de los monitoreos.

Lo invitamos a seguir cada viernes la continuación de esta investigación en: sabaconsultores.com/homicidios 


\section{REFERENCIAS}

Pande, P., Neuman, R. and Cavanagh, R. (2002). The Six Sigma way. 1st ed. New York: McGrawHill Education, pp.346-349, 355-356.

Borrego, S. (2010). Más allá de la encuesta política. 2nd ed. Monterrey: Editorial Muskaria, p. 61-70.

Gobierno de México. (2019, junio 6). Homicidios dolosos del 6/jun. Recuperado de: http:// wWw.informeseguridad.cns.gob.mx.

Montgomery, D. (2009). Introduction to Statistical Quality Control. 6th ed. Danvers, MA: Wiley, pp.180-181.

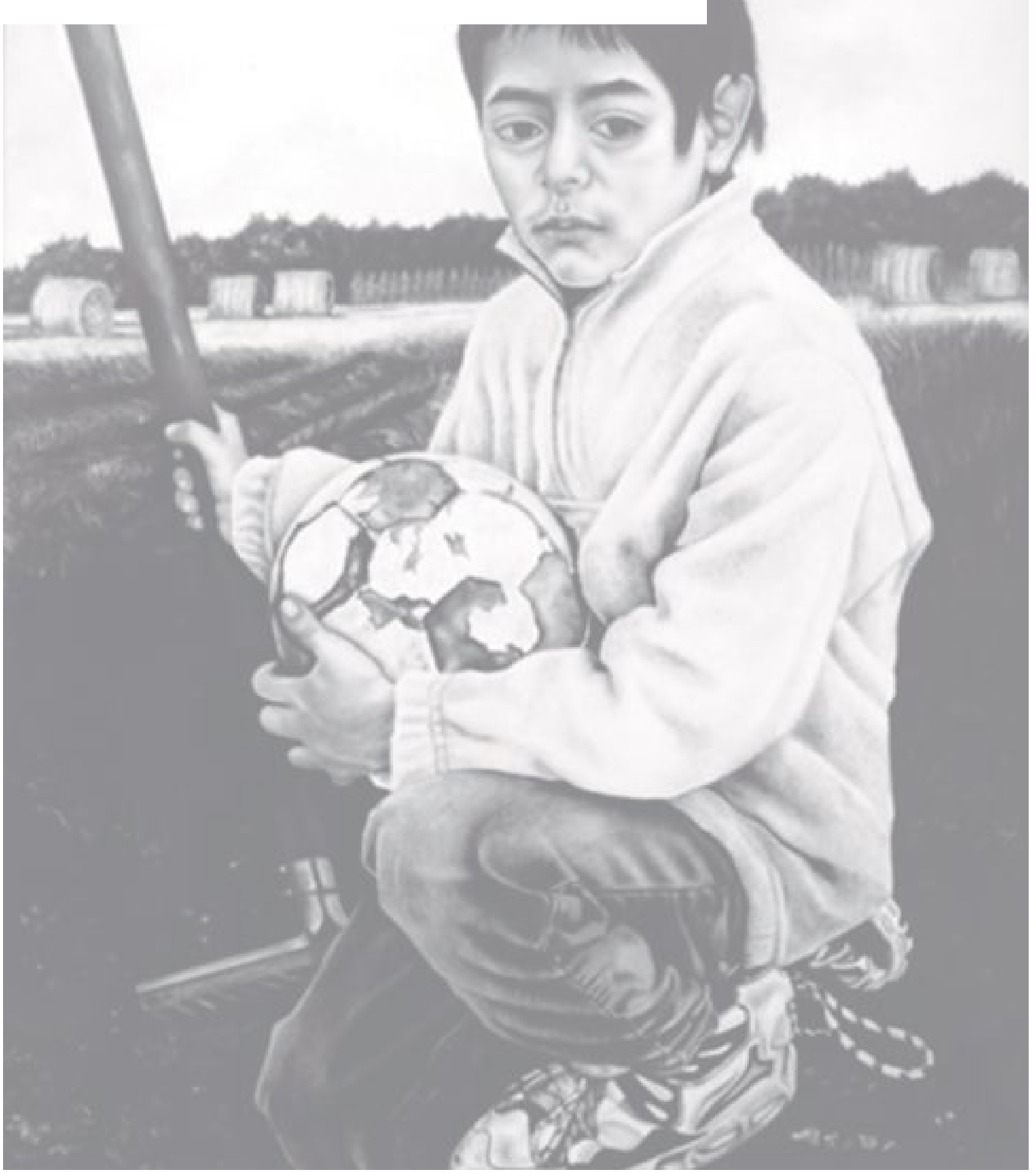




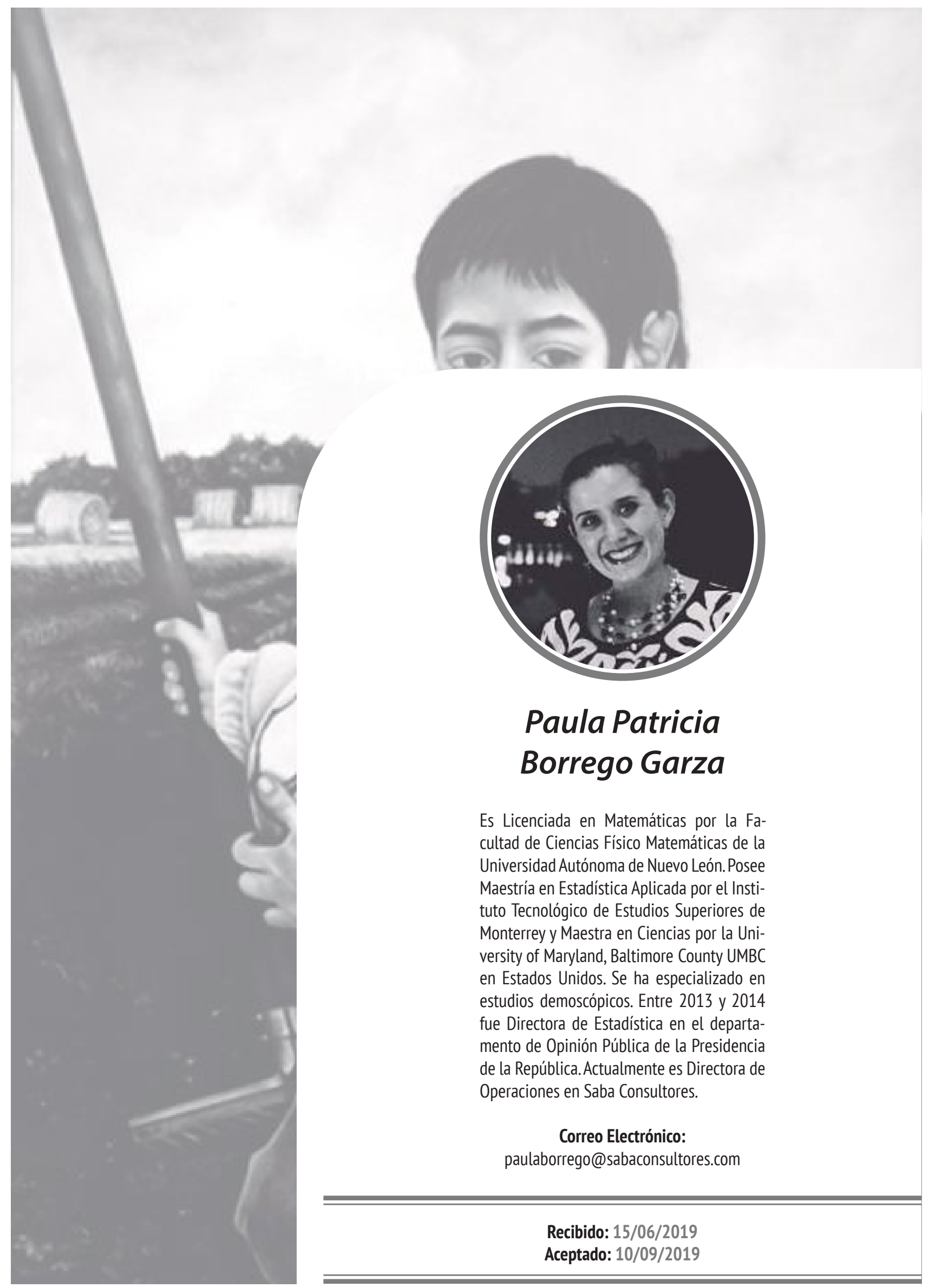

